\title{
Pregnancy: a stepping-stone to sepsis
}

\author{
Simran Sharma ${ }^{1}$, Patricia Rodrigues ${ }^{1}$, Summia Zaher $^{2}$, Luke Davies $^{1}$, and Peter Ghazal ${ }^{1}$ \\ ${ }^{1}$ Cardiff University \\ ${ }^{2}$ University Hospital of Wales Healthcare NHS Trust
}

July 28, 2021

\begin{abstract}
The physiological shifts during pregnancy predispose women to a ten-fold higher risk of developing sepsis, a life-threatening condition characterised by a maladapted host-response to infection. We present a comprehensive synthesis of maternal immunity during pregnancy, addressing whether altered set-points in immune homeostasis lower the tipping point for sepsis. This close interconnection between maternal immunity and sepsis makes clinical diagnosis highly challenging and translates to delayed antibiotics or overuse. We propose further understanding of the maternal immune set-point changes are vital for tailoring the right diagnostic tools for maternal sepsis and may unravel pathophysiological pathways that predispose an individual to sepsis.
\end{abstract}

\section{Hosted file}

Pregancy - A stepping stone to Sepsis Full manuscript with figures.pdf available at https:// authorea.com/users/428045/articles/532057-pregnancy-a-stepping-stone-to-sepsis 